\title{
Whole-cell patch-clamp recordings from identified spinal neurons in the zebrafish embryo
}

\author{
Louis Saint-Amant ${ }^{1} \&$ Pierre Drapeau ${ }^{2}$ \\ ${ }^{1}$ Department of Molecular Cellular and Developmental Biology, University of Michigan, 830 North University Ave., \\ Ann Arbor, MI, 48109-1048, USA; ${ }^{2}$ Centre for Research in Neuroscience, McGill University Health Centre Research \\ Institute, Montreal, Quebec, Canada H3G $1 A 4$
}

\begin{abstract}
We describe a preparation for obtaining patch-clamp recordings from identified embryonic spinal cord interneurons, motoneurons and sensory neurons in an in vivo zebrafish preparation. This preparation is used to study the spatial and temporal patterns of spontaneous and touch-evoked electrical activity during the initial development of circuitry
\end{abstract}

in the spinal cord. The combination of these physiological techniques with the powerful genetic and molecular tools available in the zebrafish has the potential to increase our understanding of the complex interactions between genes and electrical activity during the development of the vertebrate nervous system.

Key words: embryo, locomotion, motor behavior, neurogenesis, patch-clamp, spinal cord

\section{Introduction}

Historically, the only way to study the appearance of activity in embryonic motor networks was to look for its manifestation in the form of behaviors. Since the advent of electrophysiology it has been possible to directly study electrical activity in the networks that produce behaviors. Research in the last four decades has shown that developing neuronal networks, in both motor and non-motor areas, undergo a period of rhythmic activity that is generated independently of sensory input [1-3]. The mechanisms underlying the production of this activity are still poorly understood and it is unclear whether this activity can feedback onto cell fate determination, cell survival and synaptogenesis as the neuronal circuitry is actively forming.

Early embryonic activity patterns have been observed and studied in a variety of animal models, including the embryonic rat and mouse spinal cord. The main drawback in studying early electrical activity in mammals is that their embryonic development in utero makes recordings of spinal neurons in vivo difficult. As a result, studies in embryonic rats have relied on ventral root recordings as a measure of spinal cord activity [4, 5]. From these studies, it is unclear if activity is present in the interneuronal networks before motoneurons are able to fire action potentials or before their axons form the ventral root. Other studies have used imaging of calcium or voltage fluctuations to assess motoneuron activity in the neonatal mouse [6]. Imaging of calcium fluctuations in individual neurons can be a powerful technique to reveal neuronal involvement in population activity. One disadvantage of calcium imaging is that this method cannot provide information on key issues of synaptic connectivity, such as the mapping of synaptic connections in a network, the timing of firing between neurons in the same network, or the presence of inhibitory connections. Direct electrophysiological measurements of single and paired neurons during fictive motor activity (in paralyzed preparations) are needed to address these issues.

In recent years, the zebrafish has become an important model to study vertebrate development, partly because of the ease of working with embryos at all stages of development. Zebrafish embryos show spontaneous motor activity starting at 17 hours postfertilization. This motor activity is characterized by coils, during which the tip of the tail comes in contact with the head, the active phase of this contraction has a duration of 300 to 400 milliseconds (Saint-Amant and Drapeau, 1998). This motor behavior is transient and peaks in frequency at 19 hours $(1 \mathrm{~Hz})$. Embryos are insensitive to touch until 21 hours, at which point they start showing coils in response to touch in addition to the spontaneous coils. By 23 hours the touch response has grown in strength and is more vigorous than the spontaneous coils. The usual touch response at 1 day of development lasts longer than 800 milliseconds and is composed of an initial contralateral coil of the tail followed by 1-2 alternating coils. Embryos acquire the ability to swim in response to touch after 27 hours of development.

We have sought to use the advantages of the zebrafish preparation to study this form of early activity. This paper offers a detailed description of methods to obtain single and paired neuronal recordings in embryonic zebrafish during spontaneous and sensory evoked motor activity. Some of these 
methods have been briefly described in previous publications [8-10]. This article extends on these previous publications by providing a more thorough description of the dissection and of the techniques used for tactile stimulation and paired recordings.

\section{Materials}

Embryos and larvae are raised at $28.5{ }^{\circ} \mathrm{C}$ and are obtained from a wild type zebrafish colony maintained according to established procedures [11]. Chemicals are obtained from Sigma (Sigma Chemical Co., St. Louis, MO), Fisher (Fisher Scientific, Pittsburgh, PA) or RBI (Natick, MA). The silicone resin Sylgard is obtained from Fisher. Images of neurons are obtained with a black and white CCD camera (Panasonic BP550). Tactile stimulation of embryos is performed with a Picospritzer 2 (Parker Instrumentation). Patch-clamp and dissection electrodes are pulled from thin-walled, Kimax-51 borosilicate glass $(5 \mathrm{M} \Omega)$. Activity is recorded via an Axopatch-1D amplifier and electrodes are connected to the headstage using a HL-U holder (Axon Instruments). The data is collected on a computer with a Pentium 3 processor $(400 \mathrm{MHz}, 256 \mathrm{MB}$ RAM) running Windows 98 . The data is analyzed offline with pClamp 8 and Axoscope software (Axon Instruments). Paired recordings were obtained with current-clamp amplifiers (model BVC700 from the Dagan Corporation, Minneapolis, MN). The compound microscope used for recordings is a Leica DMLFS equipped with Hoffman modulation contrast (Leica Canada). The dissection microscope used is a Zeiss binocular scope (Zeiss Canada). The preparation is mounted on a fixed island independent of the microscope and each manipulator is mounted on a separate island. The microscope is placed on a microscope translator (Siskiyou Design Instruments) in order to move the field of view without moving the preparation or the manipulators. The micropipettes used in recordings are moved using a remotely controlled micromanipulator (Siskiyou Design Instruments).

\section{Procedures}

\section{A. Solutions}

During recordings the embryo is placed in fish artificial cerebrospinal fluid (CSF, referred to below as recording solution) modified from [12] which consisted of $(\mathrm{mM}): 134 \mathrm{NaCl}, 2.9 \mathrm{KCl}$, $2.1 \mathrm{CaCl}_{2}, 1.2 \mathrm{MgCl}_{2}, 10$ glucose, 10 Hepes, 0.015 d-tubocurarine, $290 \mathrm{mOsm}, \mathrm{pH}$ 7.8. Patchclamp electrodes (5-8 $\mathrm{M} \Omega$ resistance) are filled with a potassium gluconate solution (pipette solution) consisting of (mM): 105 potassium gluconate, $16 \mathrm{KCl}, 2 \mathrm{MgCl}_{2}, 10$ Hepes, 10 EGTA, 4
$\mathrm{Na}_{3} \mathrm{ATP}, 273$ mOsm, pH 7.2. An intracellular solution of lower osmolarity (273) than the extracellular solution (290) is used to improve seal formation [13]. All embryonic neurons are labeled during whole-cell experiments by including $0.05 \%$ SulforhodamineB in the pipette solution.

B. Dissection

Embryos ranging in age from 18 to 26 hours postfertilization (hpf) are anesthetized and paralyzed in recording solution containing $0.02 \%$ tricaine (MS-222). The embryos are pinned on their side to the bottom of a Sylgard-coated plastic petri dish by pushing short pieces of fine tungsten wire with a diameter of $25 \mu \mathrm{m}$ ( $0.001 \mathrm{in})$ through the notochord. This pinning is done under a binocular dissection scope (50× total magnification) and care is taken to avoid damaging the neighboring spinal cord (Figure 1A, dark circles). One pin is inserted at the level of the yolk sac (somites $1-3$ ) and the other one at the end of the yolk extension (somites 13-15).

To continue the dissection, the preparation is moved to a compound scope with a $40 \times$ objective (400× total magnification) equipped with Hoffman modulation optics. In order to expose the spinal cord, the skin between the two pins is removed using suction through a broken patch pipette (Figure 1A). As the skin is broken at the point of pin insertion this area is chosen to start the process of skin removal. The pipette is broken to the correct size $(\sim 25 \mu \mathrm{m})$ by flicking it across one of the tungsten pins. As a result, the diameter at the point of contact with the pin will become the size of the tip opening. Suction is used to lift the skin from the muscle underneath while the pipette is moved caudally over the trunk. The strength of suction is controlled by mouth via a syringe body, which is connected with tubing to the sideport of the electrode holder. Once the skin is removed the same pipette is used for muscle removal. At this point, collagenase (Type XI) is added to the recording solution (bath) at a final concentration of $0.02 \% \mathrm{w} / \mathrm{v}$ to facilitate muscle removal. The muscle cells overlying the spinal cord are removed from a length of 2-3 somites in a fast and repetitive rostro-caudal vacuuming motion while applying a constant suction through the pipette (Figure 1B). As the superficial muscle cells are gradually removed, the pipette is lowered to reach the deeper cells. The pipette is also moved in the dorso-ventral axis in order to remove the entire dorsal muscle mass. It is important to completely remove the muscle overlying the spinal cord as an incomplete dissection will cause some muscle cells to creep back onto the spinal cord and obscure the view of neurons. Too much suction during this procedure will damage the dura overlying the spinal cord and cause cells to be torn 


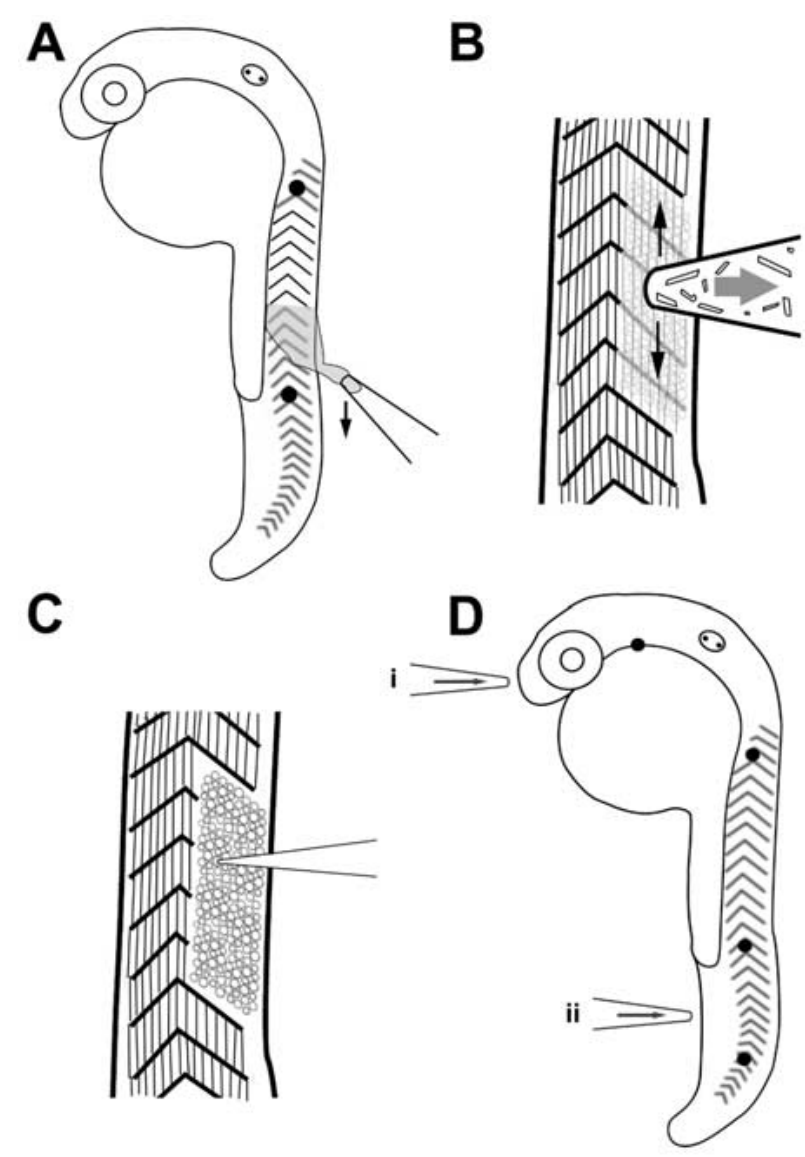

Figure 1. Pinning and dissection. $A$, tungsten pins are inserted in the notochord at the locations indicated by the dark circles. A broken patch pipette is used to remove the skin between the two pins. $B$, The muscle overlying several somites is removed by rapidly moving a broken pipette over the muscle without touching the spinal cord while applying mild suction. $C$, Once the layers of muscle are removed, the neurons are approached with a patch pipette. $D$, For stimulation protocols, extra pins are inserted caudally and rostrally. The stimulation pipette is placed either at the head (i) or the tail (ii).

from the spinal cord. Upon removal of the muscle cells, the solution with collagenase is promptly removed (usually after 5 minutes of application) by perfusing in fresh recording solution $(\sim 2$ $\mathrm{ml} / \mathrm{min}$ ). The health of the preparations is monitored by noting the transparency, and by the appearance of the neurons that have flat somata in healthy preparations but begin to round up when unhealthy. Healthy neurons are usually maintained for up to 45 minutes after the beginning of the dissection. If attempts at obtaining recordings are unsuccessful after 45 minutes, the preparation is discarded.

C. Recordings

A cell body in the spinal cord is approached while applying a small amount of positive pressure in the patch pipette. This is done until part of the transparent dura overlying the spinal cord bursts like a bubble, leaving the naked cell body exposed to the pipette (Figure 1C). The proper amount of pressure will break the dura but not flood the adjacent sections of the spinal cord with intracellular solution. The tip of the patch pipette is positioned carefully until it is pressing firmly against the cell body. Positive pressure is then released, and as a result gigaohm seals are usually obtained instantly or gently coaxed with negative pressure. In order to reach whole-cell configuration brief pulses of suction are applied to the electrode while monitoring the seal resistance. For paired recordings both pipettes are approached to the spinal cord at the same time, target neurons are quickly selected and gigaohm seals are obtained in succession. Once gigaseals are stable, attempts to reach whole-cell configuration are made on the neurons in quick succession. We use standard whole-cell recording techniques as outlined in previous publications [13] and these are performed at room temperature $\left(22^{\circ} \mathrm{C}\right)$. To insure the quality of the cells obtained, the recordings are not analyzed if the resting potential is more positive than $-40 \mathrm{mV}$ or if the input resistance is below $500 \mathrm{M} \Omega$. The average values for these parameters were $-55 \pm 2 \mathrm{mV}$ and 1400 $\pm 100 \mathrm{M} \Omega$ respectively at 24 hours of development.

D. Touch responses

Tactile stimulation of the skin is achieved by ejecting recording solution from a pipette located at the head or tail of the embryo (Figure 1D, $\sim 25-50 \mu \mathrm{m}$ tip opening). The pipette tip is placed close to the skin of the embryo before the recording pipette is approached. Recording solution fills the inside of the broken pipette by capillary action and can be pushed out by applying pressure to the pipette. The pressure (10-60 psi) and duration of the stream (10-40 ms) are precisely controlled using a Picospritzer 2 (Parker Instrumentation). In each experiment the pressure and duration are adjusted in order to find the optimal stimulation. This adjustment starts at the lowest settings (10 psi, $10 \mathrm{~ms}$ ) until the threshold is reached (50\% response rate), at which point it is increased until the response becomes consistent. The pressure will sometimes move the preparation slightly and extra pins can be added to prevent this movement (Figure 1D).

\section{Results and discussion}

The most important, and most difficult, part of this technique is the dissection to expose the spinal cord. The embryos are very fragile at this stage and learning to use the proper suction strength and shearing force during muscle removal can only be done by repeated attempts using varying amounts of force. Once mastered, a proper dissection will 
leave the dura intact and enable a clear view of the neuronal cell bodies. The visibility depends on having some type of contrast enhancement. We used Hoffman modulation optics, which works when using a plastic dish to hold the specimen. Differential interference contrast (DIC) can be used but it requires holding the specimen on a glass slide (see [14]). This visibility permits a reliable targeting of specific cell types based on soma size and soma location using both dorso-ventral and rostro-caudal coordinates. As a general rule, primary neurons $(12-16 \mu \mathrm{m})$ are much larger than secondary neurons $(6-10 \mu \mathrm{m}$; see [15-16]). In addition, there is a dorso-ventral organization of cell types in the spinal cord, from ventral motoneurons to sensory neurons at the dorsal edge, with interneurons spanning the middle of the spinal cord. Some types of neurons, such as the primary motoneurons, have stereotyped positions within each segment. In this case, the rostral primary motoneurons (RoP) are located in the middle of the somite, caudal primary motoneurons $(\mathrm{CaP})$ are located caudally near each somitic boundary, and a middle primary motoneurons (MiP) are located between the other two as the name implies (For a review of cell types in the spinal cord see [15-18]).

When a target neuron is selected the microelectrode is positioned quickly over the cell. Pushing down on the cell and moving the pipette laterally should reveal if the tip is moving the cell body directly or just the dura overlying the cell body. Positive pressure is removed only when the electrode is firmly pressing on the targeted cell. A gigaohm seal should be obtained easily with minimal suction (most of the time). If this is not the case, the shape and/or size of the pipette could be sub-optimal. When in cell-attached configuration (gigaohm seal) it is possible to detect spiking activity extracellularly. An example of this activity is seen in Figure 2A; the interburst interval and number of spikes per burst can vary depending on the cell obtained and age of the embryo. The number of spikes increase with age and primary neurons show more spiking than secondary neurons (see [9-10]).

The input resistance of many embryonic neurons is not significantly lower than the gigaohm seal resistance, therefore a drop in resistance should not be expected after the whole-cell configuration is established. One should rather look for a sudden change in membrane capacitance during a recurring depolarization step (test seal). Large capacitative transients will appear flanking the voltage steps upon establishment of whole-cell configuration. Another indication of a successful whole-cell attempt is the appearance of a sodium conductance (slower inward current spikes) in response to a recurring $30-\mathrm{mV}$ depolarizing voltage step. A stable whole-cell configuration is successfully reached in about half of the attempts. An example of a whole-cell current-clamp recording is given in Figure 2B. The whole-cell con-
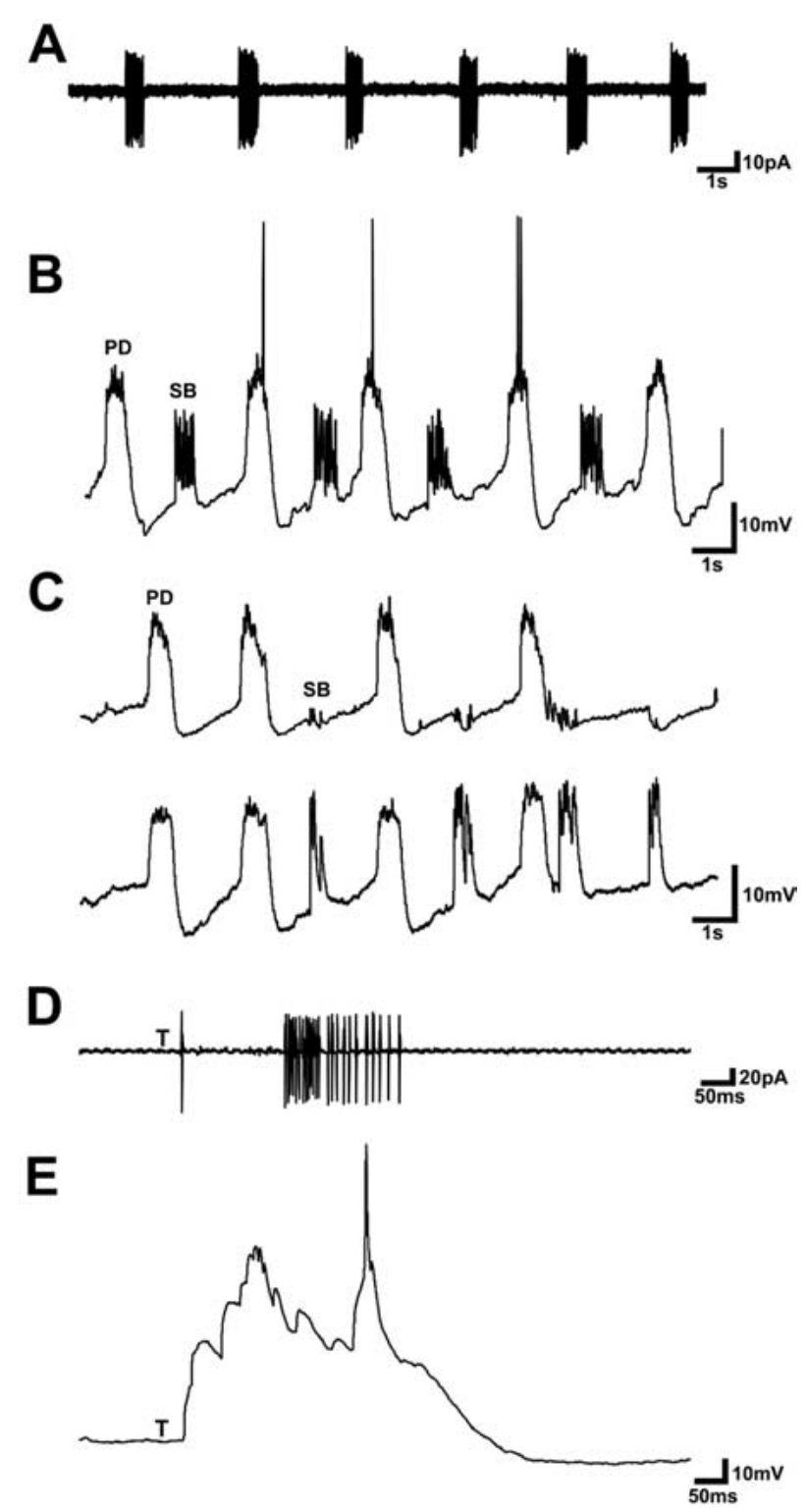

Figure 2. Sample results from motoneurons. A, Cellattached recording from a RoP motoneuron in a 22 hour old embryo. Bursts of spikes are frequent and last 300-600 ms. $B$, Whole-cell current-clamp recording from the neuron seen in $A$. Periodic depolarizations (PD) resembling short plateaus are shown, as well as long burst of synaptic activity (SB). $C$, Paired whole-cell recordings from two CaP motoneurons (somites 4, 5) at 22 hours. Coincident activity is always seen in both motoneurons. $D$, Cell-attached recording from a RoP neuron at 23 hours. The letter $\mathrm{T}$ indicates when the ipsilateral tactile stimulus was applied. In a typical response one spike is followed by a $100-200 \mathrm{~ms}$ pause in firing and subsequently by a prominent burst of spikes. $E$, Whole-cell current-clamp recording of a tactile response in a $\mathrm{CaP}$ motoneuron at 23 hours.

figuration reveals subthreshold drive to the neurons as well as inhibition. Embryonic zebrafish neurons show two main types of activity: periodic depolarization (PD) plateaus and synaptic bursts (SB). PDs are excitatory (electrical junctions, see [10]) and provide the drive for coiling contractions in the free 
moving embryos, while SBs are inhibitory (glycinergic) and prevent coincident contraction of the two sides of the trunk $[9,10]$.

The techniques outlined above for single recordings are also used for paired recordings. One electrode is approached from the dorsal side of the animal and the other from the ventral side. Overall, a successful whole-cell recording is obtained about $1 / 3$ of the time (after the protocol is optimized), which makes the probability of obtaining paired recordings less than 1/9. A particularly stable setup (vibration free) might increase the success rate for paired recordings by enabling the exchange of unsuccessful electrodes while the successful patch is maintained. Paired recordings will give information on synchronicity, as shown in Figure 2C, recordings from ipsilateral neurons always show coincident activity patterns when both the neurons are active (see [10]).

In addition to passively sampling spontaneous activity, these methods can also be used to sample sensory evoked motor responses. For example, we used tactile stimulation to evoke fictive touch responses in paralyzed embryos. Figure 2D shows a touch response in a cell-attached recording from a primary motoneuron. Figure $2 \mathrm{E}$ shows a touch response in a whole-cell recording from a primary motoneuron. The pattern of spiking in response to touch (panel D) is consistent with the normal touch response, which consists of a contralateral bend (absence of spiking in the first $200 \mathrm{~ms}$ ) followed by an ipsilateral bend (strong spiking at the end; for a full description of the behavior see [7]). The wholecell recording (panel E) reveals a strong synaptic drive at the beginning of the response when there is an absence of spiking, which is mainly a chloride conductance as determined by studying its reversal potential (L.S.A. unpublished observation). This method can be used to assess the involvement of identified neurons in evoked behaviors such as touch responses and early swimming.

When combined with other patch-clamp approaches, such as single channel recordings [14, $19,20]$ and with calcium imaging techniques [21], whole-cell patch-clamp recording can provide a detailed understanding of the cellular and synaptic mechanisms of neural network activity related to motor behaviors of the zebrafish embryo [10] and larva [22]. The usefulness of the zebrafish for forward and reverse developmental genetics is becoming very clear [18], the combination of these electrophysiological findings to the growing knowledge of zebrafish genetics should accelerate the discovery of the molecular determinants of neural network formation and function.

\section{Acknowledgments}

The authors would like to thank Mr. Wilson W. Cui, Dr. Antony W. Wood and Dr. Erica J. Crespi for helpful comments. This work was supported by a Canadian Institutes of Health Research (CIHR) Studentship to L. St.-A. and by grants from the CIHR and NSERC of Canada to P.D.

\section{References}

1. Hamburger V (1963). Some aspects of the embryology of behavior. Quat Rev Biol 38: 342-365.

2. Corner M (1964). Rhythmicity in the early swimming of anuran larvae. J Embryol Exp Morphol 12: 665-671.

3. Feller MB (1999). Spontaneous correlated activity in developing neural circuits. Neuron 22(4): 653656.

4. Kudo N, Yamada T (1987). Development of locomotor activity in the lumbar spinal cord of rat fetuses. Jap J Physiol 49: S252.

5. Nakayama K et al. (1999). Rostrocaudal progression in the development of periodic spontaneous activity in fetal rat spinal motor circuits in vitro. $J$ Neurophysiol 81: 2592-2595.

6. Branchereau P et al. (2000). Development of lumbar rhythmic networks: From embryonic to neonate locomotor-like patterns in the mouse. Brain Res Bull 53: 711-718.

7. Saint-Amant L, Drapeau P (1998). Time course of the development of motor behaviors in the zebrafish embryo. J Neurobiol 37: 622-632.

8. Drapeau $P$ et al. (1999). In vivo recording from identifiable neurons of the locomotor network in the developing zebrafish. J Neurosci Methods 88: 1-13.

9. Saint-Amant L, Drapeau P (2000). Motoneuron activity patterns related to the earliest behavior of the zebrafish embryo. J Neurosci 20: 3964-3972.

10. Saint-Amant L, Drapeau P (2001). Synchronization of an embryonic network of identified spinal interneurons solely by electrical coupling. Neuron 31 : 1035-1046.

11. Westerfield M (1995). The zebrafish book: A guide for the laboratory use of zebrafish (Brachydanio rerio). Eugene: University of Oregon Press.

12. Evans DH (1979). Fish. In: Maloiy GMO (ed.), Comparative physiology of osmoregulation in animals, 305-390. Orlando: Academic Press.

13. Hamill OP et al. (1981). Improved patch-clamp techniques for high-resolution current recording from cells and cell-free membrane patches. Pflugers Arch 391: $85-100$

14. Legendre P, Korn H (1994). Glycinergic inhibitory synaptic currents and related receptor channels in the zebrafish brain. Eur J Neurosci 6: 1544-1557.

15. Myers PZ (1985). Spinal motoneurons of the larval zebrafish. J Comp Neurol 236: 555-561.

16. Bernhardt RR et al. (1990). Identification of spinal neurons in the embryonic and larval zebrafish. J Comp Neurol 302: 603-616.

17. Andersen SSL (2001). Preparation of dissociated 
zebrafish spinal neuron cultures. Met Cell Sci 23: 205-209.

18. Drapeau P et al. (2002). Development of the locomotor network in zebrafish. Prog Neurobiol 68: 85-111.

19. Legendre P, Ali DW, Drapeau P (2000). Recovery from open channel block by acetylcholine during neuromuscular transmission in zebrafish. J Neurosci 20: 140-148.

20. Ali DW, Drapeau P, Legendre P (2000). Development of spontaneous glycinergic currents in the Mauthner neuron of the zebrafish embryo. J Neurophysiol 84 1726-1736.
21. Fetcho JR, O’Malley DM (1995). Visualization of active neural circuitry in the spinal cord of intact zebrafish. J Neurophysiol 73: 399-406.

22. Buss RR, Drapeau P (2001). Synaptic drive to motoneurons during fictive swimming in the developing zebrafish. J Neurophysiol 86: 197-210.

Author for correspondence: Louis Saint-Amant, Department of Molecular Cellular and Developmental Biology, University of Michigan, 830 North University, Ann Arbor, MI, 48109-1048, USA

Phone: 734-936-0495; Fax: 734-647-0884

E-mail: louiss@umich.edu 